\title{
Complexity of MAX-SAT Using Stochastic Algorithms
}

\author{
Mohamed Qasem \\ University of Southampton \\ Southampton, SO17 1BJ \\ United Kingdom \\ mqq06r@ecs.soton.ac.uk
}

\author{
Adam Prugel-Bennett \\ University of Southampton \\ Southampton, SO17 1BJ \\ United Kingdom \\ apb@ecs.soton.ac.uk
}

\begin{abstract}
Hill-climbing has been shown to be more effective than exhaustive search in solving satisfiability problems. Also, it has been used either by itself or in combination with other methods to solve the most difficult region of SAT, the phase transition. We show that hill-climbing also finds SAT problems difficult around the phase transition. It too follows an easyhard-eays transition.
\end{abstract}

Categories and Subject Descriptors: I.2.8 [Artificial Intelligence]: Problem Solving, Control Methods, and Search-Heuristic methods; G.3 [Probability and Statistics]: Probabilistic algorithms.

General Terms: Performance, Algorithms, Reliability.

Keywords: Satisfiability, Maximum Satisfiability, Phase Transition, Hill-climbing.

\section{INTRODUCTION}

The complexity of satisfiability (SAT) and Maximum Satisfiability (MAX-SAT) problems have been studied using complete or systematic procedures $[2,5,3,4,1]$. It has been shown that there exists a phase transition whereby the SAT follow an easy-hard-easy transition, and MAX-SAT follows an easy-hard transition. This transition occurs at clause/variable of 4.258. This has been determined empirically, and modeled by Crawford and Auton in [1]. However, the majority of phase transition experiments have been carried out using depth first search procedures. A natural question to ask is whether this phase transition exists for stochastic methods? In this paper we investigate the effect of both the number of variables and the phase transition on the performance of a simple stochastic procedure.

\section{LOCAL SEARCH PROCEDURE}

How many flips does it take to reach a local optimum? The number of flips will be obtained for a different number of variables and clauses, and the relationship between the number of flips and different parameters will be analyzed. A simple descent algorithm was used to find the local minimum, and to absolutely guarantee that a local minimum is reached an exhaustive neighborhood search was implemented.

Copyright is held by the author/owner(s).

GECCO '08 July 12-16, 2008, Atlanta, Georgia USA.

ACM 978-1-60558-131-6/08/07.

\section{EXPERIMENTAL RESULTS}

Using the fixed-clause-length model [2] random problems were generated for analysis. The number of variables used to generate our problem sets ranged from 20 to 150 variables in steps of 10 . We also varied the ratio $\alpha=m / n$ from 2.0 and 10.0 in steps of 1 . Each problem instance was searched for a local minimums with 1000 random assinments using hill-climbing and exhaustive search. Another test was run for 20,30, and 40 variables. These tests ranged from $\alpha=2$ to $\alpha=10$ in steps of 0.1 . Our first test results focused on the number of flips required to reach a local solution. Figure 1 shows the median number of flips necessary to reach a local minimum. Some values of alpha are not shown here. For these values, we were able to generate data for up to 90 variables. Beyond this point the memory requirements grew enormously. Since exhaustive search was not able to verify the local optimum we omitted these results from the plot.

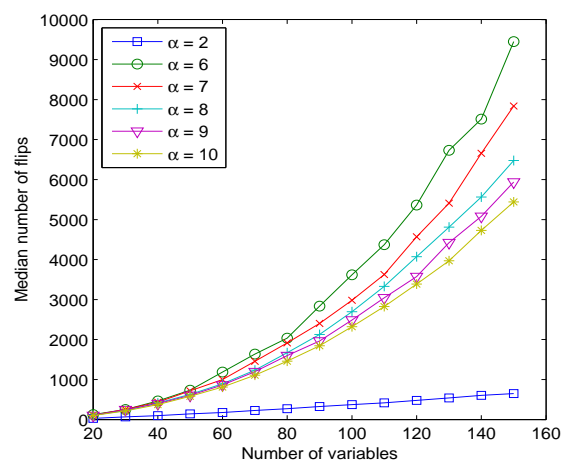

Figure 1: The median number of flips required to reach a local solution.

This is an important result since the complexity of all depth-first search methods are equivalent in that they require $2^{(n / K)}$, where $K$ is a constant, and their worst-case is asymptotically equivalent to a complete search with $2^{n}$ flips [1]. It might not be reasonable to compare two results that are different in that one, the complete procedure, finds an optimal solution while the other finds a local solution. To give more meaning to these results, another measure is required. This measure is the probability of reaching the best possible local optimum ${ }^{1}$ given a polynomial search. For 1000 runs per problem instance, we acquired the best local

\footnotetext{
${ }^{1}$ We use the term, "the best local optimum," against, "global optimum" since in most large problems it is not possible to ensure that the solution found is global.
} 


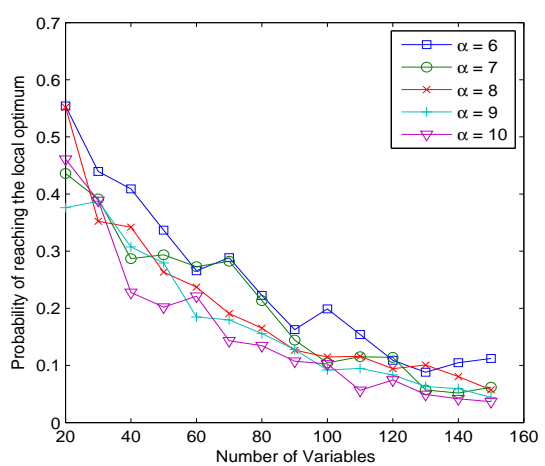

Figure 2: Probability of reaching the local optimum.

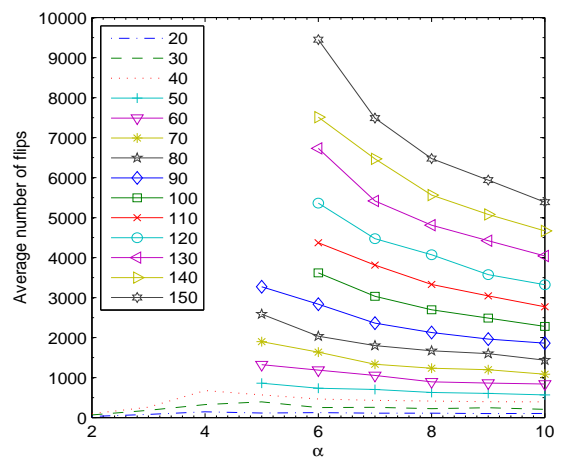

Figure 3: The median number of flips required to reach a local solution.

optimum, and determined the probability of finding it, Figure 2. The probability of getting to the best local optimum decreases as the number of variables increase and visa versa. Thus as the number of variables increase the best solution becomes harder to find.

In analyzing different clause to variable ratios, $\alpha$, it has been firmly established that at approximately $\alpha=4.3$, the random formula becomes hard specially for complete procedures $[2,5,4]$. Our results show that hill-climbing is also affected by the phase transition. The plots of the median number of flips against $\alpha$ is demonstrated in Figure 3. It is evident that around the phase transition the median number of flip increases substantially, and even more so when the number of variables becomes larger. Unfortunately, we were not able to obtain data points for 100 to 150 variables at $\alpha=5$; for the value of $\alpha=5$ the maximum we could reach was 90 variables. Even though the average number of necessary flips were not very high, yet the intense memory consumption by the exhaustive search limited the acquisition of more data points.

In another test with 20,30 and 40 variables, and varying values of $\alpha$ from 2 to 10 in increments of 0.1 with 100 clause instances per increment we obtained the graph in Figure 4. This result demonstrates the behavior of local search around the transition point. It shows that even local search finds the transition point difficult. In fact, local search for local optimums follows easy-hard-easy transition just as complete algorithms do.

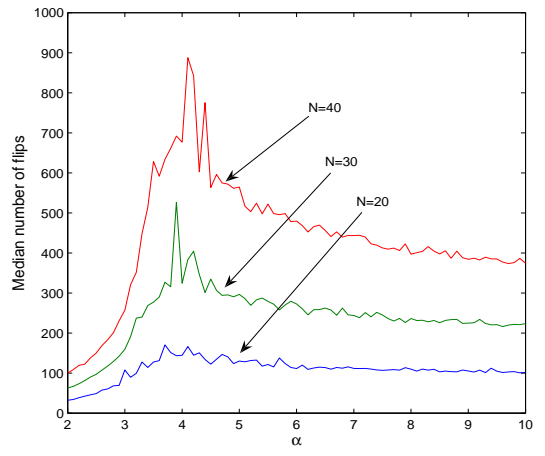

Figure 4: The median of the number of flips where plotted against $\alpha$.

Descent starts by finding global minimums very easily below the phase transition, because below this point there are large plateaus of global minimums. As $\alpha$ increase toward the phase transition, the plateaus begin to rise above the global minimums while slightly breaking up. These broken up plateaus which are still relatively large compared with global minimums present descent with more room for sideway moves before descending further (increaseing the number of flips). As $\alpha$ moves beyond the phase transition, the plateaus breakup into smaller regions creating more local minimums. Here, descent finds it easy to reach a local optimum.

\section{CONCLUSION}

We have empirically shown that a simple stochastic procedure requires a polynomial number of flips to reach a local solution in propositional satisfiability above and below the phase transition. However, the probability of finding best local optimums decreases as the number of variables increases. More significantly, stochastic methods have been extensively applied to finding solutions more efficiently especially around the transition point. In our analysis we have found that descent is influenced by the transition point in the same way complete methods are. Specifically, descent follows an easy-hard-easy transition for local optimum search.

\section{REFERENCES}

[1] J. M. Crawford and L. D. Auton. Experimental results on the crossover point in random 3-SAT. Artificial Intelligence, 81(1-2):31-57, 1996.

[2] D. Mitchell, B. Selman, and H. Levesque. Hard and easy distributions of SAT problems. pages 459-465, San Jose, CA, USA, 1992. Publ by AAAI, Menlo Park, CA, USA.

[3] R. Monasson, R. Zecchina, S. Kirkpatrick, B. Selman, and L. Troyansky. Determining computational complexity from characteristic 'phase transitions'. Nature, 400(6740):133-7, 1999.

[4] B. Selman. Stochastic search and phase transitions: AI meets physics. volume vol.1 of IJCAI-95. Proceedings of the Fourteenth International Joint Conference on Artificial Intelligence, pages 998-1002, Montreal, Que., Canada, 1995. Morgan Kaufmann Publishers.

[5] Z. Weixiong. Phase transitions and backbones of 3-SAT and maximum 3-SAT. Principles and Practice of Constraint Programming - CP 2002. 7th International Conference, CP 2001. Proceedings (Lecture Notes in Computer Science Vol.2239), pages 153-67, Paphos, Cyprus, 2001. Springer-Verlag. 